\title{
Chemical exfoliation and optical characterization of threading- dislocation-free gallium-nitride ultrathin nanomembranes
}

\author{
Rami T. ElAfandy ${ }^{a}$, Mohammed A. Majid ${ }^{a}$, Tien Khee Ng${ }^{a}$, Lan Zhao ${ }^{b}$, Dongkyu Cha ${ }^{\mathrm{b}}$, Boon S.
} $\mathrm{Ooi}^{\mathrm{a}}$

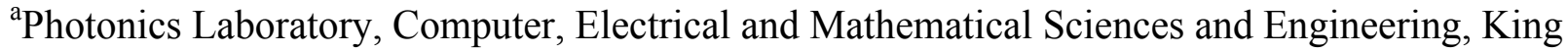
Abdullah University of Science and Technology (KAUST), Thuwal, Kingdom of Saudi Arabia 23955-6900; ${ }^{\mathrm{b}}$ Core Lab of Imaging and Characterization, King Abdullah University of Science and Technology (KAUST), Thuwal, Kingdom of Saudi Arabia 23955-6900

\begin{abstract}
Semiconductor nanostructures have generated tremendous scientific interests as well as practical applications stemming from the engineering of low dimensional physics phenomena. Unlike 0D and 1D nanostructures, such as quantum dots and nanowires, respectively, 2D structures, such as nanomembranes, are unrivalled in their scalability for high yield manufacture and are less challenging in handling with the current transfer techniques. Furthermore, due to their planar geometry, nanomembranes are compatible with the current complementary metal oxide semiconductor (CMOS) technology. Due to these superior characteristics, there are currently different techniques in exfoliating nanomembranes with different crystallinities, thicknesses and compositions. In this work we demonstrate a new facile technique of exfoliating gallium nitride $(\mathrm{GaN})$ nanomembranes with novel features, namely with the non-radiative cores of their threading-dislocations (TDs) being etched away. The exfoliation process is based on engineering the gallium vacancy $\left(\mathrm{V}_{\mathrm{Ga}}\right)$ density during the $\mathrm{GaN}$ epitaxial growth with subsequent preferential etching. Based on scanning and transmission electron microscopies, as well as micro-photoluminescence measurements, a model is proposed to uncover the physical processes underlying the formation of the nanomembranes. Raman measurements are also performed to reveal the internal strain within the nanomembranes. After transferring these freely suspended $25 \mathrm{~nm}$ thin $\mathrm{GaN}$ nanomembranes to other substrates, we demonstrate the temperature dependence of their bandgap by photoluminescence technique, in order to shed light on the internal carrier dynamics.
\end{abstract}

Keywords: gallium nitride, nanomembrane, single crystalline, threading dislocation, electroless etching, nanostructure, transmission electron microscope, Raman spectroscopy

\section{INTRODUCTION}

There is currently a growing scientific and technological interest in single crystalline gallium nitride $(\mathrm{GaN})$ material due to its wide bandgap energy $(3.4 \mathrm{eV})$, high saturation carrier velocity $\left(2.5 \times 10^{7} \mathrm{~cm} . \mathrm{s}^{-1}\right)$ and high breakdown field $\left(3 \mathrm{MV} . \mathrm{cm}^{-1}\right) .{ }^{1} \mathrm{GaN}$ based devices have ushered the way for several staggering technologies including solid state lighting, high-density optical data storage, high power electronics and laser based projectors and TVs. ${ }^{2,3} \mathrm{~A}$ new class of $\mathrm{GaN}$ devices based on nanomembranes has lately been emerging. These devices take advantage of the high crystalline quality, and thus high optical efficiencies, of the nanomembranes combined with their two dimensional low flexural rigidity. For example, nanomembrane field effect transistors (FETs) ${ }^{4}$ and light emitting diodes (LEDs) ${ }^{5}$ have been successfully demonstrated having electrical and optical properties similar to their bulk counterparts but operating on flexible substrates. There are currently several demonstrated techniques for the exfoliation of $\mathrm{GaN}$ nanomembranes, each tacking advantage of a different $\mathrm{GaN}$ property. The main technique used to exfoliate the $\mathrm{GaN}$ nanomembranes relies on growing a sacrificial layer (or bulk) buried underneath a thin layer of GaN with subsequent chemical etching of the sacrificial layer. Mei et al., exfoliated GaN nanomembrane by epitaxially growing GaN on silicon with subsequent etching of silicon by a mixture of nitric and hydrofluoric acids. ${ }^{6}$ Park et al., exfoliated p-doped $\mathrm{GaN}$ from a n-doped $\mathrm{GaN}$ sacrificial layer through doping selective photo-electrochemical etching. ${ }^{7}$ Stonas et al., buried an InGaN sacrificial layer under $\mathrm{GaN}$ and subsequently etched it through bandgap selective photo-electroless etching. ${ }^{8}$ A different technique, demonstrated by Tiginyanu et al., relies on charging the 
surface of a GaN wafer through argon ion bombardment in order to make (only) the surface resistant to photoelectroless etching. ${ }^{9}$ Yerino et al., developed another technique which does not rely on a buried sacrificial layer, by using anodic electrochemical etching with a varying potential bias to form undersurface buried cavities. ${ }^{10}$ Subsequent annealing in nitrogen/ammonia causes the cavities to merge together which allows the surface to get exfoliated. Here, we demonstrate a new technique for exfoliating GaN nanomembranes with novel characteristics, namely, the non-radiative cores of their threading dislocations (TDs) being etched away. ${ }^{11}$ The process is believed to depend on vertical variations of the gallium vacancy $\left(\mathrm{V}_{\mathrm{Ga}}\right)$ density induced during epitaxial growth.

\section{EXPERIMENT}

The GaN wafers used in this study consisted of $30 \mu \mathrm{m}$ of silicon doped $\left(\sim 10^{18} \mathrm{~cm}^{-3}\right)$ c-plane oriented GaN grown using hydride vapor phase epitaxy (HVPE) technique on a sapphire substrate. The wafers have an average TD density of $10^{8} \mathrm{~cm}^{-2}$. During the top 20-30 nm GaN, the growth temperature has decreased. The GaN wafers are then cleaved into $1 \mathrm{~cm}^{2}$ pieces which are later degreased in acetone and isopropanol alcohol (IPA) and finally cleaned in hot $\mathrm{HNO}_{3}\left(65^{\circ} \mathrm{C}\right)$. Using a reactive ion sputtering system, a thin layer of platinum $(150 \mathrm{~nm})$ is deposited on the side of the samples to act as a cathode. The samples are then placed in a bath containing $\mathrm{CH}_{3} \mathrm{OH}: \mathrm{H}_{2} \mathrm{O}_{2}(35 \%): \mathrm{HF}(48 \%)$ with the top surface (c-plane) in contact with the solution (Figure 1). A fused silica lens is used to focus ultraviolet (UV) radiation, emanating from a $200 \mathrm{~W}$ mercury $(\mathrm{Hg})$ arc lamp, onto the sample surface. Once the desired etching period is reached, the samples are cleaned by dipping and rinsing in IPA and then dried using a critical point dryer (CPD).

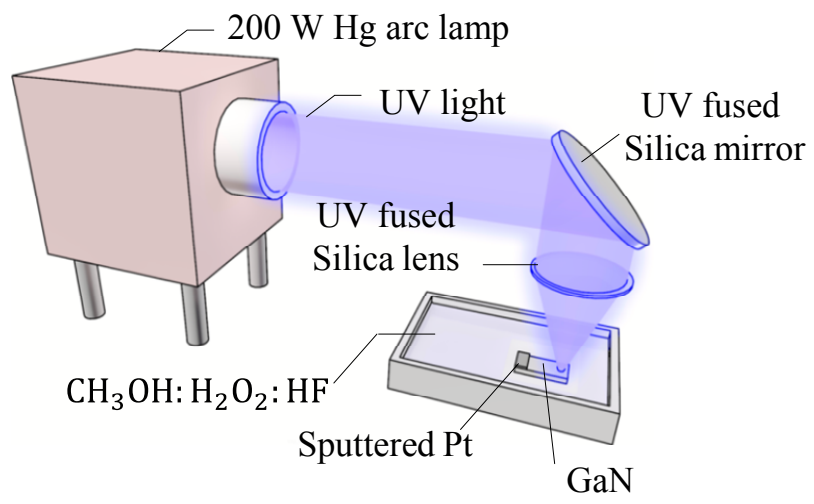

Figure 1. The experimental setup of UV-assisted electroless chemical etching for the exfoliation of the GaN nanomembranes.

\section{RESULTS AND DISCUSSION}

\subsection{Exfoliation model}

The etching process depends on the presence of a surface charge region (SCR) at the n-GaN/electrolyte interface arising due to the surface fermi-level equilibrium with the solution electrochemical potential $(\tilde{\mu}){ }^{12}$ The SCR is characterized by the presence of surface electric fields which cause an upward bending in the GaN surface energy bands. UV assisted electroless etching initiates when an incident photon generate an electron hole pair. If the pairs are generated up to the hole diffusion length away from the SCR, holes may diffuse to the SCR where they drift to the interface electric fields causing the oxidation of $\mathrm{GaN}$ to $\mathrm{Ga}_{2} \mathrm{O}_{3}$ which is later etched away by $\mathrm{HF}$ (Figure 2(a)). ${ }^{13,14}$ The presence of gallium vacancies $\left(\mathrm{V}_{\mathrm{Ga}}\right)$, which act as deep acceptors, will compensate the Si n-doping and therefore decrease the bending within the surface bands, causing a decrease in the etching rate (Figure 2(b)). ${ }^{15}$ The process described here is believed to originate from a varying $\mathrm{V}_{\mathrm{Ga}}$ density within the GaN during growth by manipulating the growth temperature. Growing GaN at high temperature (HT-GaN), results in a high quality GaN with low $\mathrm{V}_{\mathrm{Ga}}$ density. Switching to low growth temperature (LT-GaN) typically results in an increase in $\mathrm{V}_{\mathrm{Ga}}$ density, as well as a reduction in Ga incorporation on the $\{10 \overline{1} 1\}$ which causes a decrease in GaN growth on the TD terminations on the surface. ${ }^{16,17}$ Thus, it can be inferred that the growth of tens of nanometers of LT-GaN on top of 
HT-GaN will form a layer void of TDs'cores and contain an increased $\mathrm{V}_{\mathrm{Ga}}$ density. Thus, once etched, the top surface will have a slower etching rate than the underneath layer.
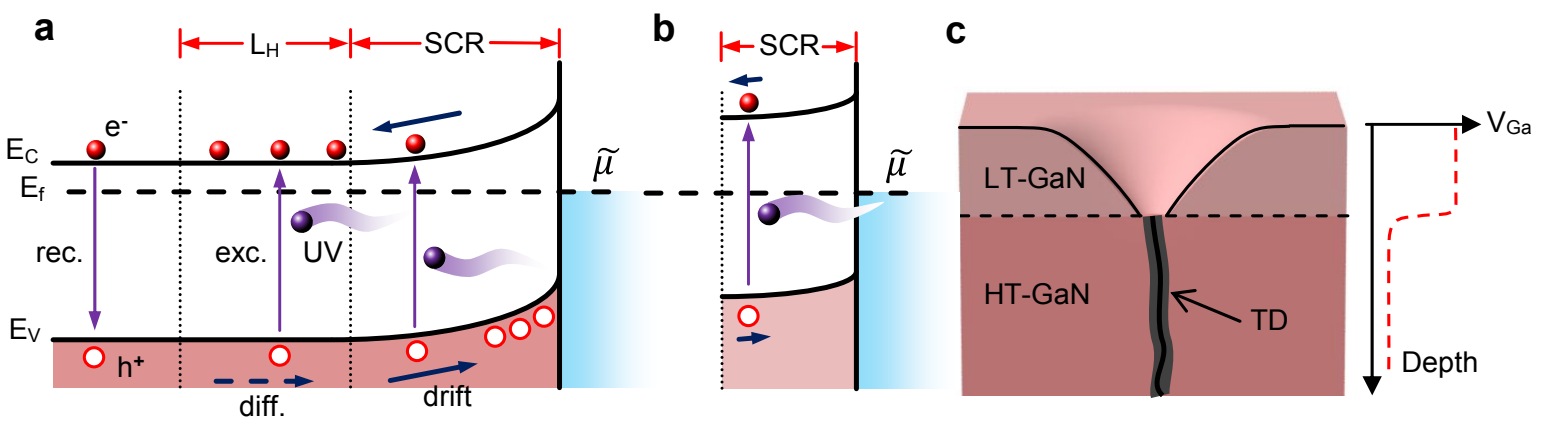

Figure 2. Energy band diagrams for the case of (a) a high temperature growth (HT-GaN), low $\mathrm{V}_{\mathrm{Ga}}$ density GaN and (b) a low temperature growth (LT-GaN), high $\mathrm{V}_{\mathrm{Ga}}$ density GaN.

\subsection{Exfoliation process}

The etching process is illustrated, step-by-step, by means of the SEM images in Figure 3. The etching solution starts attacking the GaN surface at regions where TDs terminate on the surface, due to the presence of high band bending, which causes the formation of surface pores (Figure 3 (a)). The etching proceeds along the [000 $\overline{1}]$ crystallographic direction (dashed arrow in Figure 3(b)). At around $25 \mathrm{~nm}$ deep from the surface, just under the LT-GaN, the etching proceeds horizontally as well. As the lateral etching proceeds, more pores begin to nucleate and propagate along the [000 $\overline{1}$ ] crystallographic direction causing the formation of an undersurface pore domain (Figure 3 (c)). ${ }^{18,19}$ As the pore domains grow under the surface, they start merging (Figure $3(\mathrm{~d})$ ) and the top un-etched surface becomes only supported by few columns (Figure 3 (e)). Eventually the columns get etched away causing the formation of a freely suspended 20-30 nm thin GaN nanomembrane (Figure 4(a)). After the exfoliation of the nanomembrane, the pore domains become exposed. Figure 4(b) shows a pore domain has been outlined and the primary pores marked.
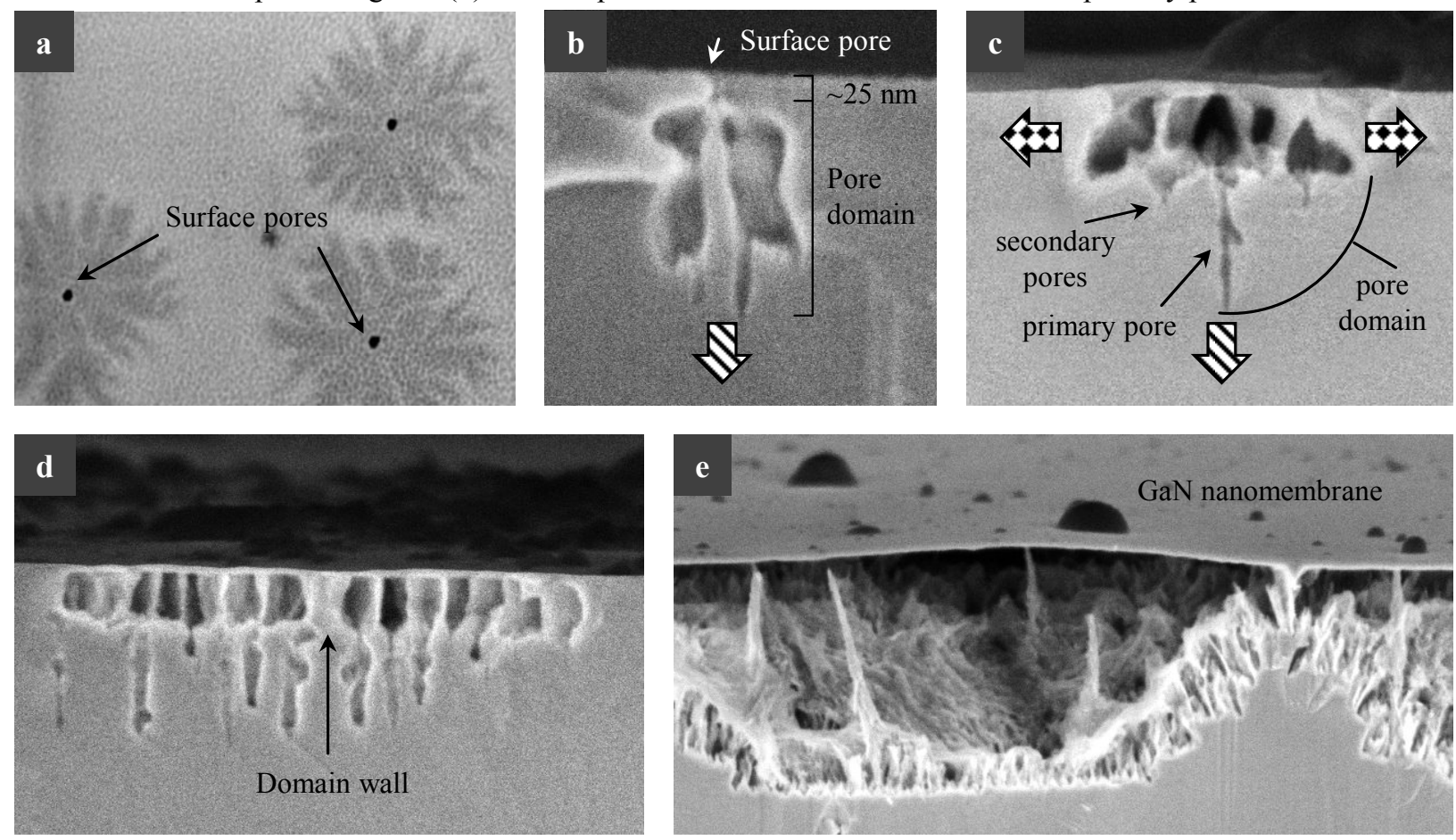

Figure 3. SEM images taken at different stages of the exfoliation process of the GaN nanomembrane. (a) top and (b) side view of a pore domain with the top surface pores. (c) The initial growth of a pore domain. (d) The beginning of merging of two pore domains. (e) Local formation of a nanomembrane. 

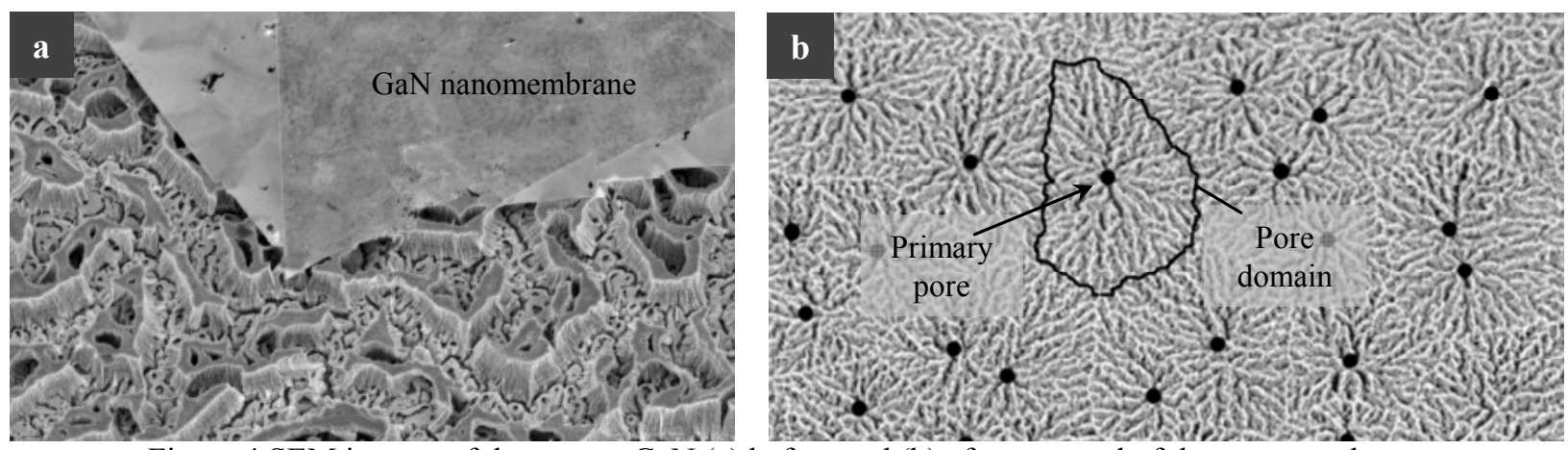

Figure 4 SEM images of the porous GaN (a) before and (b) after removal of the nanomembrane.

Nanowires (NWs), or whiskers, are common to form in UV-assisted electroless etching from TDs' cores which offer fast recombination channels for the electron-hole pairs and thus deplete the surrounding region from holes. Through careful examination of the SEM image in Figure 5 (a), the NW tips, formed from the TD cores, appear exactly under the surface pores. Interestingly, upon drying without using a CPD, the nanomembrane collapses downward under the effect of the water surface tension causing the NWs to protrude through the nanomembrane pores that are present on top of them ( Figure 5(b,d)). Since the surface pores lie exactly above the NWs, are formed from TD cores, the nanomembrane has to be void of these non-radiative TD cores.
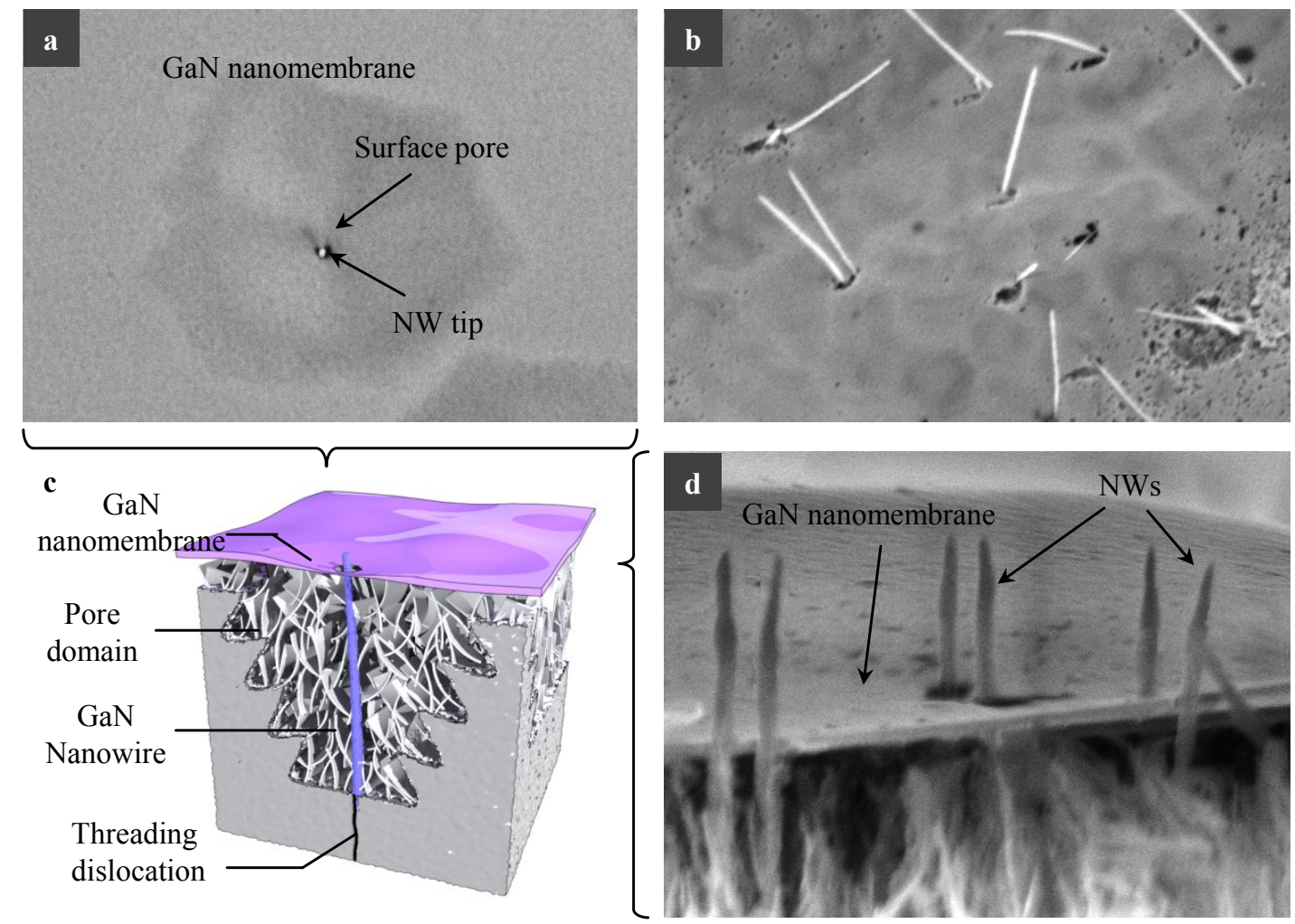

Figure 5. (a) top, (b) inclined and (d) side views SEM images of the NWs forming underneath the surface pores of the nanomembrane. (c) Schematic representation of a pore domain forming underneath a nanomembrane with the nanowire forming from TD at the center of the pore domain. 


\subsection{Transmission electron microscope analysis}

Figure 6(a) is a cross sectional TEM imaging of a single pore domain. The top layer clearly shows a nanomembrane locally formed on top of a pore domain. The image reveals a TD running through the center of the pore domain as previously described through the proposed model. Figure 6(b) clearly shows the cross sectional TEM image of the $\mathrm{GaN}$ nanomembrane formed on top of the pore domain. Since the crystalline nature of the NM highly affects its physical (thermal, electrical and optical) properties, a small piece of the NM is mechanically transferred, by means of a $40 \mu \mathrm{m}$ tungsten probe to a carbon coated copper grid for TEM imaging. Through high resolution TEM (HRTEM) of the nanomembrane, with imaging axis aligned to the c-plane, presented in Figure 6(c), the high degree of crystallinity of the nanomembrane is clearly observed.
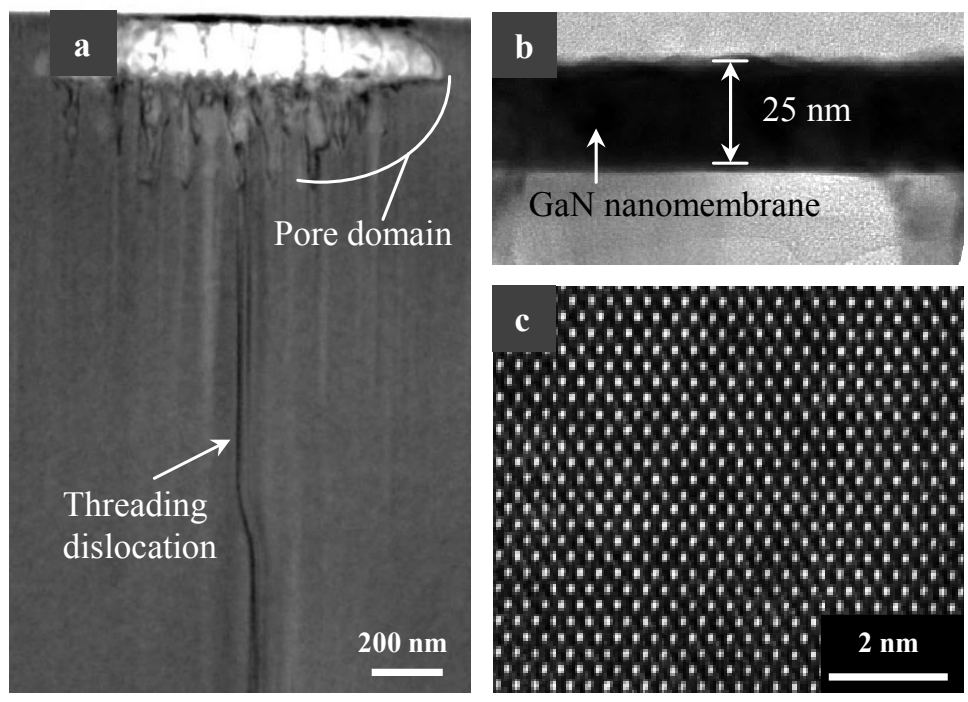

Figure 6. Transmission electron microscopy images of the GaN nanomembrane showing (a) TD running through the center of the pore domain, (b) cross sectional TEM image of the GaN nanomembrane formed on top of the pore domain, and (c) high resolution TEM (HRTEM) of the nanomembrane showing high degree of crystallinity of the nanomembrane.

\subsection{Optical characterization}

After analyzing the crystalline nature of the GaN nanomembranes using TEM, we further characterize their optical properties. In order to eliminate any emission from the bulk GaN underneath the nanomembrane, an approximately 100 um wide nanomembrane is transferred onto sapphire prior to photoluminescence measurements (inset Figure 7).

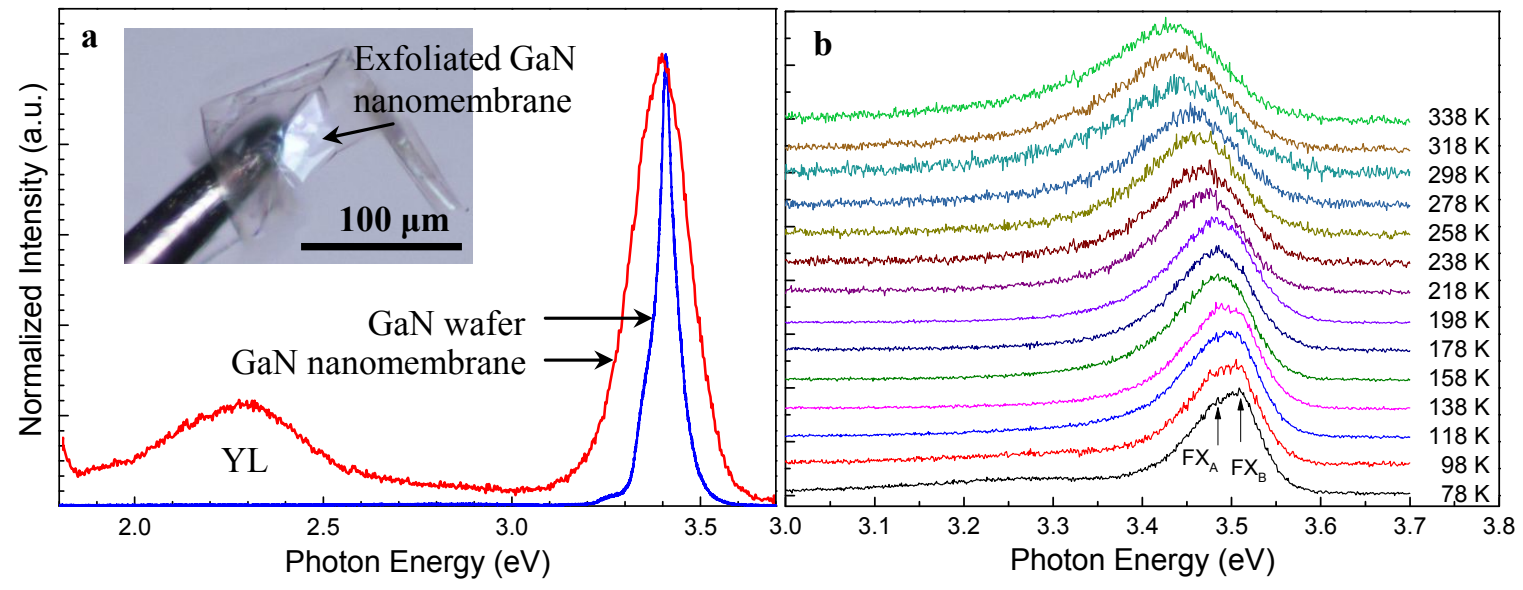

Figure 7. Photoluminescence emission from the GaN nanomembrane at (a) room temperature, and (b) variation of the photoluminescecnce spectra from $77 \mathrm{~K}-338 \mathrm{~K}$. 
Using a $325 \mathrm{~nm} \mathrm{He}-\mathrm{Cd}$ laser, at room temperature, photoluminescence emissions are measured from a GaN wafer (prior to the etching process) and from an exfoliated and transferred nanomembrane. While both the near bandgap emissions are approximately centered around $3.4 \mathrm{eV}$, the emission from $\mathrm{V}_{\mathrm{Ga}}$ (yellow luminescence at $2.4 \mathrm{eV}$ ) is more pronounced in the nanomembrane emission than the wafer emission. The near bandgap emission from the nanomembrane is then measured at different temperatures from $78 \mathrm{~K}$ to $338 \mathrm{~K}$. As expected, a clear broadening and redshift is apparent during the heating process. While at $78 \mathrm{~K}$, two peaks can be observed which are ascribed to free excitons A and B, they are hardly resolved due to their wide full width at half maximum (FWHM). This is probably due to the lower temperature growth of the NM, and hence the increase in $V_{G a}$, which has decreased the crystal quality.

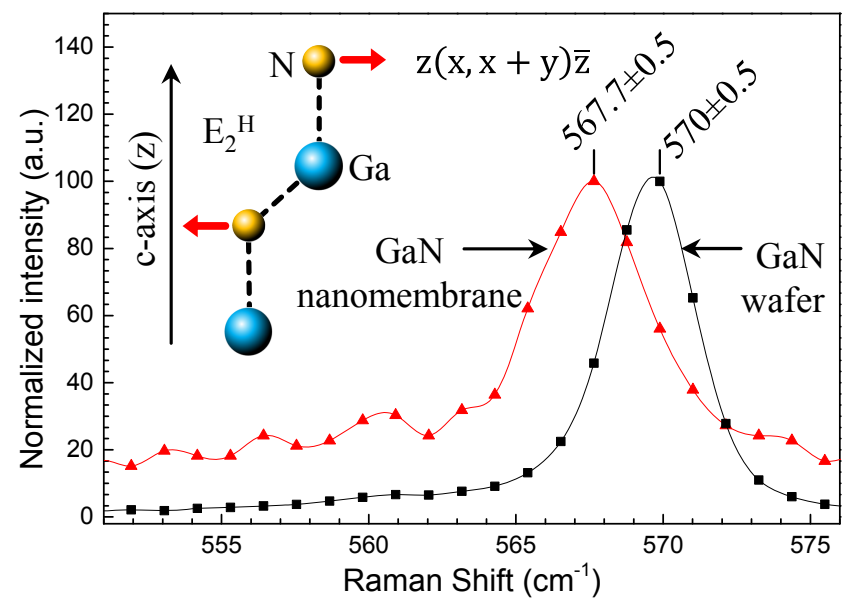

Figure 8. Raman shift of the $\mathrm{E}_{2}{ }^{\mathrm{H}}$ phonon mode of the $\mathrm{GaN}$ nanomembrane and wafer (GaN on sapphire).

A unique property of nanomembranes based devices, as compared to the bulk, is the ability to relax the internal strain within the structure. The first order Raman signal is collected under the $\mathrm{z}(\mathrm{x}, \mathrm{x}+\mathrm{y}) \bar{z}$ scattering configuration causing the $E_{H}^{2}$ and the $E_{L}^{2}$, the high and low frequency branches of the E2, respectively, and the A1(LO) to become the only allowed optical phonon modes during the measurement. The $\mathrm{E}_{2}{ }^{\mathrm{H}}$ Raman signals from a GaN wafer (prior to etching) and from an exfoliate nanomembrane are plotted in Figure 8. While the Raman signal from the bulk GaN (on sapphire) demonstrates a higher Raman shift due to the residual biaxial compressive strain from the sapphire substrate, the Raman signal from the exfoliate nanomembrane is at the zero strain vibrational frequency. ${ }^{20}$ Thus, once the nanomembrane is exfoliated from the wafer, the crystal is relaxed and any residual compressive strain vanishes.

\section{CONCLUSION}

We have demonstrated a new technique for exfoliating $\mathrm{GaN}$ nanomembranes based on depth variation of the $\mathrm{V}_{\mathrm{Ga}}$ density during growth followed by UV-assisted electroless etching. The exfoliated nanomembranes have the nonradiative cores of the TDs etched away during the exfoliation process. Through SEM images, we have demonstrated the etching/exfoliation process step by step. TEM imaging has been employed to shed light onto the crystalline structure of the nanomembranes. Finally, the optical properties of the exfoliated and transferred nanomembranes have been analyzed through studying their photoluminescence emission and their Raman signals. The presented process can be further be engineered in terms of varying the growth conditions (doping compositions, LT-GaN thickness, growth temperature) in order to exfoliate membranes of different thicknesses and lateral dimensions. 


\section{ACKNOWLEDGMENTS}

Rami T. ElAfandy gratefully acknowledges the financial support from KAUST baseline funding and KACST Technology Innovation Center for Solid State Lighting at KAUST.

\section{REFERENCES}

[1] Chow, T. P. and Tyagi, R., "Wide bandgap compound semiconductors for superior high-voltage unipolar power devices," Electron Devices, IEEE Transactions on, vol. 41, pp. 1481-1483, (1994).

[2] Ponce, F. and Bour, D., "Nitride-based semiconductors for blue and green light-emitting devices," Nature, vol. 386, pp. 351-359, (1997).

[3] Chichibu, S. F., Uedono, A., Onuma, T., Haskell, B. A., Chakraborty, A., Koyama, T., Fini, P. T, Keller, S., DenBaars, S. P., Speck, J. S., Mishra, U. K., Nakamura, S., Yamaguchi, S., Kamiyama, S., Amano, H., Akasaki, I., Han, J., Sota, T., "Origin of defect-insensitive emission probability in In-containing (Al, In, Ga) N alloy semiconductors," Nature materials, vol. 5, pp. 810-816, (2006).

[4] Xiong, K., Park, S. H., Song, J., Yuan, G., Chen, D., Leung, B., Han, J., "Single Crystal Gallium Nitride Nanomembrane Photoconductor and Field Effect Transistor," Advanced Functional Materials, (2014).

[5] Park, S. H., Yuan, G., Chen, D., Xiong, K., Song, J., Leung, B., Han, J., "Wide Bandgap III-Nitride Nanomembranes for Optoelectronic Applications," Nano Letters, vol. 14, pp. 4293-4298, (2014).

[6] Mei, Y., Thurmer, D. J., Deneke, C., Kiravittaya, S., Chen, Y.-F., Dadgar, A., Bertram, F., Bastek, B., Krost, A., Christen, J. r., Reindl, T., Stoffel, M., Coric, E., Schmidt, O. G., "Fabrication, Self-Assembly, and Properties of Ultrathin AlN/GaN Porous Crystalline Nanomembranes: Tubes, Spirals, and Curved Sheets," ACS Nano, vol. 3, pp. 1663-1668, (2009).

[7] Park, J., Song, K. M., Jeon, S.-R., Baek, J. H., Ryu, S.-W., "Doping selective lateral electrochemical etching of GaN for chemical lift-off," Applied Physics Letters, vol. 94, (2009).

[8] Stonas, A. R., T Margalith, T., DenBaars, S. P., Coldren, L. A, Hu, E. L., "Development of selective lateral photoelectrochemical etching of InGaN/GaN for lift-off applications," Applied Physics Letters, vol. 78, p. 1945, (2001).

[9] Tiginyanu, I., Popa, V., Stevens-Kalceff, M. A., "Membrane-assisted revelation of the spatial nanoarchitecture of dislocation networks," Materials Letters, vol. 65, pp. 360-362, (2011).

[10] Yerino, C. D., Zhang, Y., Leung, B., Lee, M. L., Hsu, T.-C., Wang, C.-K., Peng, W.-C., Han,, "Shape transformation of nanoporous GaN by annealing: From buried cavities to nanomembranes," Applied Physics Letters, vol. 98, (2011).

[11] ElAfandy, R. T., Majid, M. A., Ng, T. K., Zhao, L., Cha, D., Ooi, B. S., "Exfoliation of Threading Dislocation-Free, Single-Crystalline, Ultrathin Gallium Nitride Nanomembranes," Advanced Functional Materials, vol. 24, pp. 2305-2311, (2014).

[12] Rajeshwar, K., "Fundamentals of semiconductor electrochemistry and photoelectrochemistry," Encyclopedia of electrochemistry, (2007).

[13] Youtsey, C., Bulman, G., Adesida, I., "Dopant-selective photoenhanced wet etching of GaN," Journal of electronic materials, vol. 27, pp. 282-287, (1998).

[14] Vajpeyi, A., Tripathy, S., Chua, S., Fitzgerald, E., "Investigation of optical properties of nanoporous GaN films," Physica E: Low-dimensional Systems and Nanostructures, vol. 28, pp. 141-149, (2005).

[15] Neugebauer, J., and Walle, C. G. V. d., "Gallium vacancies and the yellow luminescence in GaN," Applied Physics Letters, vol. 69, pp. 503-505, (1996).

[16] Wu, X. H., Elsass, C. R., Abare, A., Mack, M., Keller, S., Petroff, P. M., DenBaars, S. P., Speck, J. S., Rosner, S. J., "Structural origin of V-defects and correlation with localized excitonic centers in InGaN/GaN multiple quantum wells," Applied Physics Letters, vol. 72, pp. 692-694, (1998).

[17] Mahanty, S., Hao, M., Sugahara, T., Fareed, R. S. Q., Morishima, Y., Naoi, Y., Wang, T., Sakai, S., "Vshaped defects in InGaN/GaN multiquantum wells," Materials Letters, vol. 41, pp. 67-71, 10// (1999).

[18] Erne, B., D. Vanmaekelbergh, D., Kelly, J., "Morphology and strongly enhanced photoresponse of GaP electrodes made porous by anodic etching," Journal of the Electrochemical Society, vol. 143, pp. 305-314, (1996). 
[19] O’Dwyer, C., Buckley, D., Sutton, D., Serantoni, M., "An Investigation by AFM and TEM of the Mechanism of Anodic Formation of Nanoporosity in n-InP in KOH," Journal of The Electrochemical Society, vol. 154, pp. H78-H85, (2007).

[20] Perlin, P., Jauberthie-Carillon, C., Itie, J. P., San Miguel, A., Grzegory, I., Polian, A., "Raman scattering and x-ray-absorption spectroscopy in gallium nitride under high pressure," Physical Review B, vol. 45, pp. 83-89, (1992). 